\title{
The nasal response to exercise and exercise induced bronchoconstriction in normal and asthmatic subjects
}

\author{
KINGMAN P STROHL, MICHAEL J DECKER, LESLIE G OLSON, TOD A FLAK, \\ PETER L HOEKJE
}

Airway Disease Center, Departments of Medicine, University Hospitals of Cleveland; and Case Western Reserve University, Cleveland, Ohio, USA

ABSTRACT Two studies were carried out to test the hypothesis that the fall and recovery of nasab resistance after exercise in asthmatic and non-asthmatic subjects are related to the development og bronchoconstriction after exercise. In study 1 nasal resistance (posterior rhinomanometry) and specific airway resistance (sRaw) were measured before challenge and one, five, 10 and 30 minute after four minutes of exhausting legwork exercise in nine asthmatic subjects and nine age matche healthy subjects. One minute after exercise there was a reduction in nasal resistance of $49 \%$ (SD $15 \%$ from baseline in the healthy subjects and of $66 \%(17 \%)$ in the asthmatic subjects. This response and the subsequent return of nasal resistance to baseline values did not differ significantly between the tw $\overrightarrow{80}$ groups despite a substantial difference in the change in sRaw, an increase of $74 \%(45 \%)$ in the asthmatic subjects 10 minutes after exercise, and no change in the non-asthmatic subjects. In study 20 nasal and specific airway resistances were monitored according to the same measurement protocol in six subjects with increased airway reactivity. Subjects exercised on two occasions, wearing a noseclip

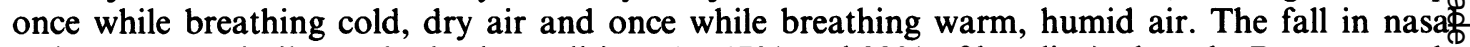
resistance was similar under both conditions (to $47 \%$ and $39 \%$ of baseline), though sRaw rose onle after cold air inhalation (to $172 \%$ of baseline). The results indicate that the nasal response to exercise is not related to bronchial obstruction in asthmatic subjects after exercise or to the temperature of humidity of the air inspired through the mouth during exercise.

\section{Introduction}

The nasal response to exercise could be altered by airway or chest wall neural reflexes evoked by exercise induced airway obstruction. Studies in anaesthetised animals have shown that vagal reflexes influence nasal patency.' As autonomic dysfunction may be a feature of airway reactivity, ${ }^{23}$ changes in autonomic activity might have different effects on the nasal vasculature volume in those who have asthma following exertion. This is supported by the findings of Syabbalo et l $^{4}{ }^{4}$ who examined subjects with asthma and allergic rhinitis and found that the rate of return of nasal resistance to baseline after exercise was altered by bronchoconstriction.

We have carried out two studies to examine whether exercise induced bronchoconstriction could alter the

Address for reprint requests: Dr Kingman P Strohl, University Hospitals, Airway Disease Center, 2074 Abington Road, Cleveland, Ohio, 44106, USA.

Accepted 27 July 1988 nasal responses to exercise. In the first (study 1) the time course of change in nasal resistance in response to exercise was measured in age matched groups of healthy and asthmatic subjects. In the second (study 2 . similar measurements were performed; the condition of the air inspired via the mouth during exercise was however, changed so that the subsequent bronchial response to exercise in each subject could be altered $\frac{D}{2}$

\section{Methods}

\section{SUBJECTS}

Nine healthy, normal subjects (six male) and 12 asthmatic subjects (six male) participated in the twe studies. All subjects gave written informed conseff before the study, as laid down by the Institutiona Review Board for Human Investigation, University Hospitals of Cleveland. The asthmatic subjects had previous diagnosis of asthma and a history of episodit wheezing or post-exertional wheezing (or both). ${ }^{5}$ Thes were in a stable condition and could refrain from taking their medications for at least six and usually ( $1 \Phi$ 


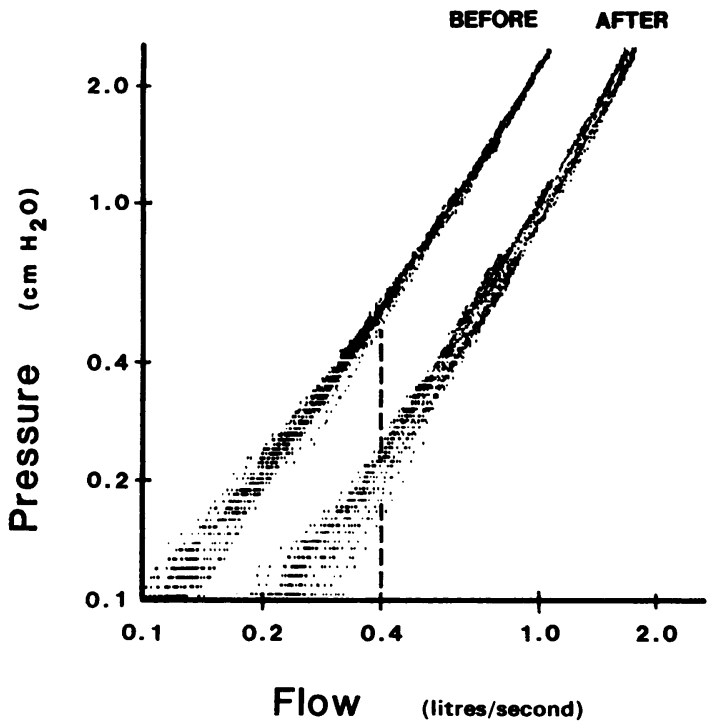

Fig 1 Natural logarithmic plots of inspiratory flow versus transnasal pressure for one healthy subject before and one minute after four minutes of exercise. The dashed vertical line extends from the logarithmic value of 0.4 litres a second. With exercise there is a parallel shift of the relationship to the right, indicating enlargement of the nasal passage.

of 12 asthmatics) 12 hours before being studied. Healthy subjects were not taking any medication at the time of study. One healthy subject and six asthmatic subjects had a history of allergic rhinitis. Before each study forced expiratory volume in one second (FEV $)_{1}$ and functional residual capacity (FRC) were measured and the results compared with predicted values. ${ }^{67}$ Healthy subjects had an FRC of $85-105 \%$ of their predicted value. The asthmatic subjects had an FEV 1 greater than $70 \%$ predicted and an FRC less than $135 \%$ of predicted.

\section{NASAL RESISTANCE}

Transnasal pressure and bulk flow were measured by posterior rhinomanometry. ${ }^{8}$ Pressure and flow were displayed on the $x$ and $y$ axes of a storage oscilloscope (Tektronix 5A18N) and recorded in scalar form (Graphtec Linearcorder, Mark IV WR3101) and digitally $(1000 \mathrm{~Hz})$ with an IBM XT microcomputer. Data were recorded for 20 seconds, during which the subject made inspiratory and expiratory efforts of varying magnitude through the nose at a rate of 0.2-2 Hz. Subjects were initially coached to achieve equal flow on inspiration and expiration, even during maximal efforts. Data were, however, collected from submaximal efforts at flows where no flow limitation occurred.

The pressure-flow data were analysed by plotting inspiratory values of $\ln$ flow against $\ln$ pressure $^{89}$; in such a plot changes in the pressure-flow relationships associated with exercise are seen as a parallel shift in the $\ln$-ln plot (fig 1). We used the pressure at an inspiratory flow rate of 0.4 litres a second to describe the position of the ln pressure-ln flow relationship.

\section{SPECIFIC AIR WAY RESISTANCE}

Specific airway resistance was measured by body plethysmography, while the subject was seated, breathing through the mouth and wearing noseclips. The product of thoracic gas volume and specific airway resistance (specific resistance: sRaw) was computed. Five measurements of sRaw were obtained on each occasion and the result reported is the average of these values.

\section{PROTOCOLS}

All subjects were in the laboratory at least 30 minutes before baseline tests were performed. Baseline measurements of nasal resistance and $F E V_{\text {, were made }}$ before and after measurements of FRC and sRaw. Body plethysmography did not affect nasal pressureflow measurements in any subject, and the mean of the two control values for nasal resistance was obtained. Measurements of nasal and specific airway resistance were repeated at one, five, 10 , and 30 minutes after exercise.

To determine whether the study had any effect on physiological measurements, a subgroup of six healthy and three asthmatic subjects went through the protocol without exercising. There were no significant changes in sRaw or in nasal resistance in these circumstances.

\section{Study 1}

We measured nasal and airway resistance before and one, five, 10 , and 30 minutes after exercise in nine healthy (Nos 1-9) and nine asthmatic (Nos 10-18) subjects. The age range was $20-41$ years for healthy subjects and 20-42 years for asthmatic subjects. The exercise task was four minutes on a bicycle ergometer (Pedalmate, WE Collins) at a work rate that produced about $60 \%$ of the predicted maximal oxygen consumption for that subject. ${ }^{10}$ Exercise was performed without constraining the route of airflow and while the subject was breathing room air $\left(20-23^{\circ} \mathrm{C}, 30-40 \%\right.$ relative humidity). Subjects were able to breathe through either the mouth or the nose. With brief exercise, at these workloads, however, they generally breathed through their mouth."

\section{Study 2}

Using the same protocol and exercise task, we measured nasal and specific airway resistance in five asthmatic (Nos 15, 19-21) subjects and one healthy subject (No 6). The asthmatic subjects were chosen 
because they had good lung function $\left(\mathrm{FEV}_{1}\right.$ and FRC $>85 \%$ predicted). The healthy subject was known to have enhanced bronchial reactivity, responding to exercise, while breathing cold air, with a $9 \%$ fall in $\mathrm{FEV}_{1}$. Subjects exercised while wearing a noseclip and breathed only through the mouth. On one occasion the subjects inspired cold, dry air and on the other they inspired warm, humidified air. Conditioned air was generated by heat exchangers. ${ }^{12}$ With cold air challenge temperatures at the mouthpiece were $-0^{\circ} \mathrm{C}$ at $10 \mathrm{l} / \mathrm{min}$ and $-9^{\circ} \mathrm{C}$ at $40 \mathrm{l} / \mathrm{min}$, and relative humidity was below $5 \%$. With warm air challenge temperatures were $32^{\circ} \mathrm{C}$ at $10 \mathrm{l} / \mathrm{min}$ and $39.5^{\circ} \mathrm{C}$ at $40 \mathrm{l} /$ min, and relative humidity was $95-97 \%$. Five subjects exercised under both conditions on the same day, the tests being separated by 90 minutes. One subject exercised under each condition but on separate days. The order of conditions was warm-cold $(n=4)$ or cold-warm $(n=2)$.

\section{STATISTICS}

Results for each group are expressed as means with standard deviations in parentheses. Comparisons between groups (study 1) and between challenges (study 2) were analysed by means of multivariate analysis of variance on all measurements with a repeated measures design. The criterion for statistical significance was $p<0.05$. Data on the recovery of nasal resistance were analysed for each individual to give the slope of changes in nasal resistance over time (1-30 min after exercise) and an intercept of resistance with the time axis was extrapolated on the basis of values from one to 30 minutes after exercise.

\section{Results*}

\section{Study 1}

Baseline FEV 1 and FRC were $97 \%$ (SD 5\%) and 95\% (4\%) of predicted for the healthy subjects, and $84 \%$ $(10 \%)$ and $109 \%(8 \%)$ of predicted for the asthmatic subjects ( $p<0.05$ for both). The exercise task was 916 (323) kilopond meters/min ( $\mathrm{kpm} / \mathrm{min})$ in the healthy subjects and $752(213) \mathrm{kpm} / \mathrm{min}$ in the asthmatic subjects. Baseline and post-exercise values of nasal resistance and sRaw are shown in figure 2. Baseline nasal resistance, at a pressure of $0.41 / \mathrm{s}$, was $1.27(0.47)$ $\mathrm{cm} \mathrm{H}_{2} \mathrm{O} / 1 . \mathrm{s}$ in the healthy subjects and $6.21(1.70) \mathrm{cm}$ $\mathrm{H}_{2} \mathrm{O} / \mathrm{l}$.s in the asthmatic subjects $(\mathrm{p}<0.05)$. The corresponding baseline measurements of specific airway resistance (sRaw) were $4.76(0.41) \mathrm{cm} \mathrm{H}_{2} \mathrm{O} / \mathrm{s}$ and $8.59(3.06) \mathrm{cm} \mathrm{H}_{2} \mathrm{O} / \mathrm{s}$ respectively. The difference in nasal resistance and sRaw between the healthy and

\footnotetext{
*Conversion: Traditional to SI units-Nasal airway resistance: $1 \mathrm{~cm}$ $\mathrm{H}_{2} \mathrm{O} / 1 . \mathrm{s}=0.098 \mathrm{kPa} / \mathrm{l} . \mathrm{s}$; specific airway resistance: $1 \mathrm{~cm} \mathrm{H}_{2} \mathrm{O} . \mathrm{s}$ $=0.098 \mathrm{kPa} . s ;$ power (exercise task): $1 \mathrm{kpm} / \mathrm{min}=0.164$ watts $=0.164 \mathrm{~J} / \mathrm{s}$.
}

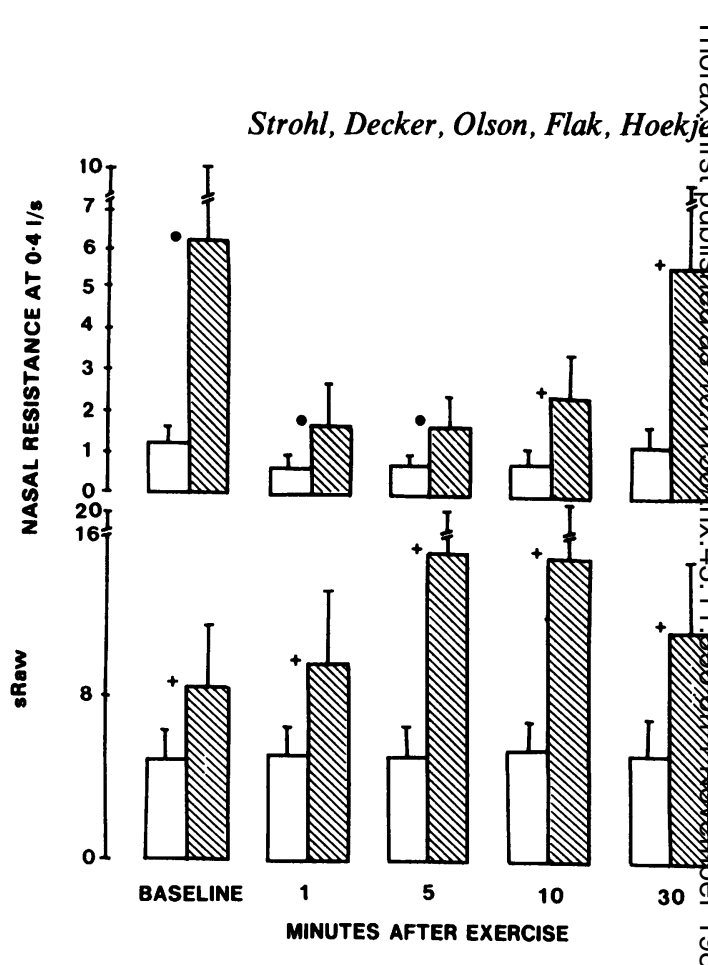

Fig 2 Protocol 1: (grouped values (mean and SD)) for nasal resistance and airway resistance (sRaw) before (baseline) and at the specified times after exercise. Open bar represent values from healthy subjects and hatched bars values from asthmatic subjects. $+p<0.01, \bullet p<0.05$ in the comparison with baseline values.

asthmatic subjects were significant at each time oह̂ measurement.

Exercise caused no significant change in sRaw in the healthy subjects; a significant fall in nasal resistance was, however, seen one minute after exercise $(49 \%$ $(15 \%)$ of baseline). This was relatively unchanged five and 10 minutes after exercise (fig 3 ) but had returned to baseline values by 30 minutes.

In the asthmatic subjects exercise resulted in significant increase in sRaw five minutes after exercise $(171 \%(45 \%)$ of baseline; $p<0.01)$; the increase was maintained five and 10 minutes after exercise but was less at 30 minutes (fig 3). There was a significant fall in nasal resistance beginning one minute after exercise $(66 \%(17 \%)$ from baseline; $p<0.01)$, and this slowly returned to baseline between five and 30 minutes aftes exercise.

Although the percentage change in sRaw waS significantly higher in the healthy subjects five, 10 , and 30 minutes after exercise, the fall in nasal resistance्e after exercise did not differ significantly between the two groups. Nasal resistance had returned to baseline values 30 minutes after exercise in both groups. The mean slope of recovery in nasal resistance and the extrapolated intercept showed large intersubjec $\mathbb{B}$ differences in both groups, particularly the asthmatic 


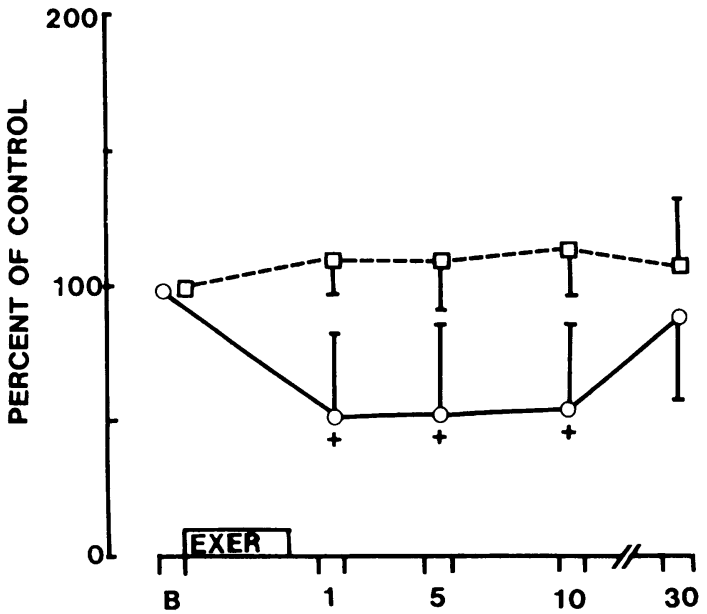

MINUTES AFTER EXERCISE

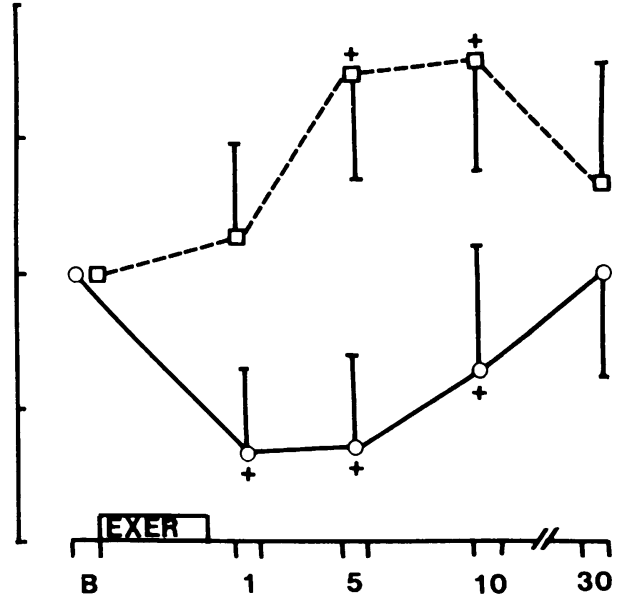

MINUTES AFTER EXERCISE

Fig 3 Protocol 1: Changes, expressed as percentages (mean and SD) of baseline values, in nasal resistance at $0.4 \mathrm{l} / \mathrm{s}$ (circles) and specific airway resistance (squares) before (B) and at the specified times after exercise. Values for healthy subjects are on the left and for asthmatic subjects on the right. $+p<0.01$ in the comparison with baseline values.
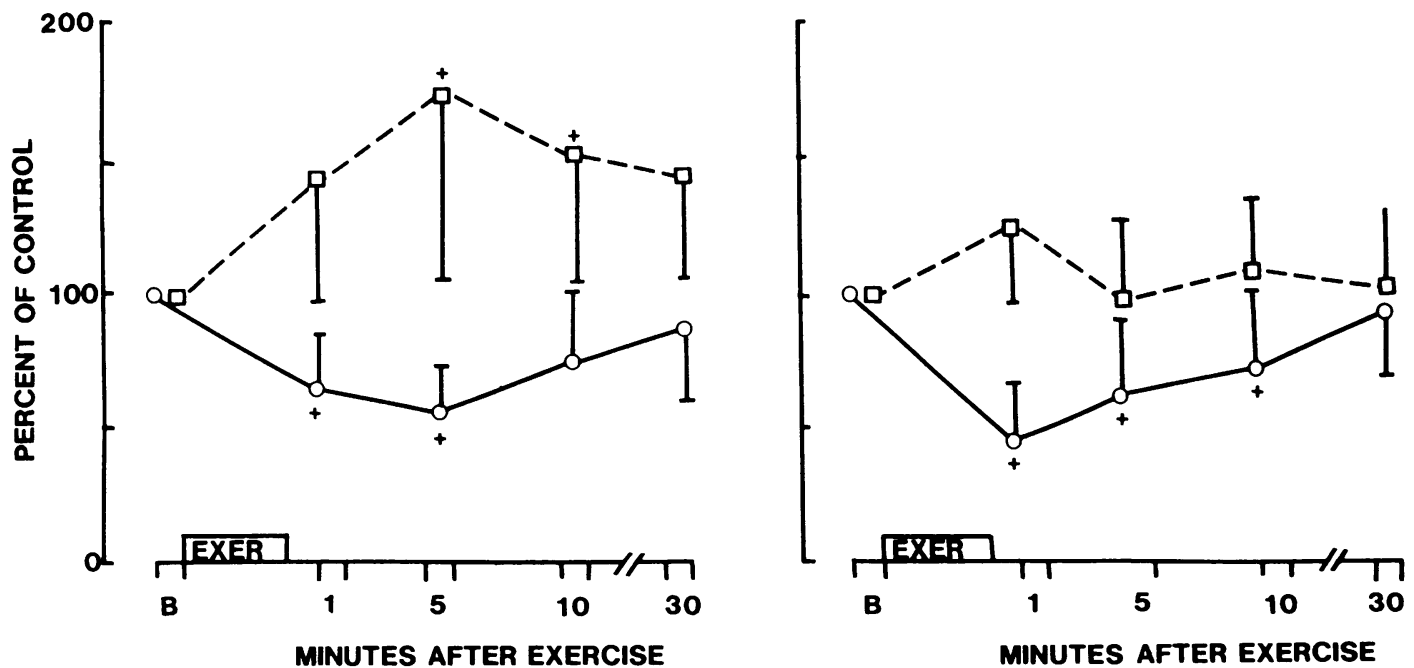

Fig 4 Protocol 2: Changes, expressed as percentages (mean and SD) of baseline (control) values, in nasal resistance at $0.4 \mathrm{l} / \mathrm{s}$ (circles) and specific airway resistance (squares) before (B) and at the specified times after exercise. Results from trials using cold air during exercise are shown on the left and results from trials using warm air on the right.

$+p<0.01$. 
group, and no significant difference between the groups.

\section{Study 2}

FEV ${ }_{1}$ and FRC for the six subjects in study 2 were $91 \%$ (SD 4\%) and $102 \%(3 \%)$ of predicted. Baseline nasal resistance was $2.60(1.11) \mathrm{cm} \mathrm{H}_{2} \mathrm{O} / 1 . s$ before exercise with cold air and $2.53(0.87) \mathrm{cm} \mathrm{H}_{2} \mathrm{O} / 1$.s before exercise with warm, humid air $(\mathrm{p}=0.07)$. Values of sRaw were also similar, $4.69(0.88)$ and $5.39(1.41) \mathrm{cm} \mathrm{H}_{2} \mathrm{O} / \mathrm{s}$ respectively. The exercise task for each individual was identical under the two conditions, the mean workload of $858(297) \mathrm{kpm} / \mathrm{min}$ representing $53 \%(12 \%)$ of predicted maximal oxygen consumption. Results from the subject without a history of asthma were within the ranges observed in the five asthmatic subjects, so results were pooled.

sRaw was unchanged when exercise was performed with the subjects inspiring warm, humid air (fig 4). It rose when the subject exercised breathing cold air to $172 \%(84 \%)$ of baseline at five minutes and to $152 \%$ $(57 \%)$ of baseline at 10 minutes. Nasal resistance fell in both studies, by $47 \%(20 \%)$ one minute after exercise with cold air and by $39 \%(24 \%)$ after exercise with warm air. There was no significant difference between cold and warm air in the maximum reduction in nasal resistance or the fall one, five, and 10 minutes after exercise or in the slope or extrapolated intercept of recovery in nasal resistance.

\section{Discussion}

These results show that the nasal response to exercise occurs irrespective of the bronchoconstrictor response to exercise. Several factors might have contributed to this finding. The asthmatic subjects required higher transnasal pressures than the healthy subjects to achieve the same flow, possibly because four of the asthmatic patients had a history of allergic rhinitis. This could also account for the higher variability in the nasal response in asthmatic subjects, though it did not cause a significant difference in the change in nasal resistance with exercise between the two groups.

Syabbalo et $\mathrm{al}^{4}$ have examined the effect of exercise on the nose and lower airways in subjects who did and did not develop bronchoconstriction in response to exercise. They used different methods to assess the healthy and asthmatic subjects, however. Healthy subjects performed exercise on a cycle ergometer and their airway response was measured as change in $\mathrm{FEV}_{1}$; the asthmatic subjects performed treadmill exercise and their airway response was measured as change in peak expiratory flow (PEF). The nasal response in their study was measured in the same way in the two groups, as the transnasal pressure that developed with a constant flow through the nose of
$0.4 \mathrm{l} / \mathrm{s}$. They found that post-exercise recovery in nasasp resistance occurred more quickly in subjects wh developed bronchoconstriction in response to exercise than in those who did not. Our experimental methods and exercise task were similar for the two groups iळ study 1 and we found little systematic difference्s between the nasal responses in the two groups.

There are no obvious clinical or physiological factors that could have obscured a difference between the asthmatic and healthy subjects. The similarits cannot be accounted for by an overlap in the history of allergic rhinitis or atopy or in nasal resistance. It could represent the effects of competing autonomic reflexes (sympathetic outflow to nose and parasympatheti $\overrightarrow{\varepsilon_{0}}$ output to the airways), but there is little evidence thaf exercise induced bronchoconstriction results from reflexes from the exercise task as such. ${ }^{1314}$ Exercise induced humoral influences could have resulted in the similar nasal responses. Richerson and Seebohm, however, showed that blockade of the cervical sym pathetic activity in man blocked the fall in nasao resistance, suggesting that circulating substances $d \sigma^{\mathbb{D}}$ not cause the fall in nasal resistance in response to $\overrightarrow{0}$ exercise in healthy subjects. The nasal responses inpo asthmatic and healthy subjects may have a different basis, but this is unlikely.

In study 2 we examined the nasal response to an identical exercise task performed under two differen conditions of inspired air, designed respectively toD provoke and to attenuate exercise induced broncho constriction. ${ }^{3}$ Control values for nasal and airway resistance differed from those in study 1 . Subjects were chosen on the basis of good baseline lung function an known airway sensitivity to cold air challenge, which accounts for difference in control values. Subjectड wore a noseclip and breathed through a mouthpiecei while exercising. The use of noseclips during exercise has an insignificant effect on the nasal response to exercise $^{8}$; it did, however, give the opportunity to alteg the conditions of inspired air during exercise and modify the subsequent bronchial response. ${ }^{12}$ As expeco ted ${ }^{12-14}$ exercise while the subject was breathing cold dry air caused bronchoconstriction whereas exercises with the subject breathing warm, humid air did not The magnitude and time course of changes in nasal resistance with exercise, however, were similar regard t less of the condition of inspired air during exercise and regardless of whether airway narrowing occurred ${ }_{\sigma}^{\omega}$ These results suggest that bronchoconstriction following exercise does not modify the time course of changes in nasal resistance. Vagally mediated reflex? effects, proprioceptive reflexes from the chest wall, or cardiovascular eflects of bronchoconstriction are therefore unlikely to have a major influence on nasa $\vec{D}$ resistance under these conditions.

As the fall in nasal resistance with exercise was 
uninfluenced by the condition of inspired air, pharyngeal, laryngeal, and tracheal reflexes ${ }^{15}$ evoked by changes in temperature or humidity of the inspired air are also unlikely to influence the nasal response to exercise. Breathing cold air through the nose is known to result in nasal congestion, a response thought to aid in the heating and humidification of air through the nose. ${ }^{16}$

Our results indicate that asthmatic subjects have a nasal response to exercise similar to that of healthy subjects. As neither bronchoconstriction after exercise nor thermal conditions of the airstream in the mouth, pharynx, and trachea during exercise altered the pattern of change in nasal function after exercise, exercise is likely to have caused a sympathomimetic reduction in airway resistance both in healthy and in asthmatic subjects, with the bronchial response to exercise predominantly a local phenomenon.

We thank Dr E R McFadden Jr for his encouragement and advice. We are also grateful for the statistical consultation provided by Dr Pamela Byard. This work is supported in part by the National Institutes of Health (HL29726, HL33791) and a Specialized Center of Research grant (HL37117) and by the Cystic Fibrosis Foundation (Rainbow Chapter) of Ohio. KPS holds a Research Career Development Award (HL02011), and LGO was a travelling scholar of the Royal Australasian College of Physicians.

\section{References}

1 Eccles KS, Eccles R. Nasal vasodilation induced by electrical stimulation of the vagus. Rhinology 1981; 20:89-92.

2 Barnes PJ, Dollery CT, MacDermot J. Increased pulmonary alpha-adrenergic and reduced beta-adrenergic receptors in experimental asthma. Nature 1980;285: 569-71.

3 Karliner M, Shelhamer J, Smith L, et al. Autonomic abnormalities and allergy. Ann Intern Med 1982;96: 349-57.

4 Syabbalo NC, Bundgaard A, Widdicombe JG. Effects of exercise on nasal airflow resistance in healthy subjects and in patients with asthma and rhinitis. Bull Eur Physiopathol Respir 1985;21:507-13.

5 American Thoracic Society. Definition and classification of chronic bronchitis, asthma, and pulmonary emphysema. Am Rev Respir Dis 1980;85:762-9.

6 Knudson RJ, Slatin RC, Lebowitz MD, Burrows B. The maximal expiratory flow volume curve. Normal standards, variability, and effects of age. Am Rev Respir Dis 1976;113:587-600.

7 Cotes JE. Lung function: assessment and application in medicine. 4th ed. Oxford: Blackwell, 1979.

8 Olson LG, Strohl KP. The response of the nasal airway to exercise. Am Rev Respir Dis 1987;135:356-9.

9 Richerson HB, Seebohm PM. Nasal airway response to exercise. J Allergy 1968;41:269-84.

10 Jones NL, Campbell EJM. Clinical exercise testing. 2nd ed. Philadelphia: Saunders, 1982:118.

11 Niinimaa V, Cole P, Mintz S, Shepard RJ. The switching point from nasal to oronasal breathing. Respir Physiol 1980;42:61-71.

12 McFadden ER Jr, Lenner KA, Strohl KP. Postexertional airway rewarming and thermallyinduced asthma: new insights into pathophysiology and possible pathogenesis. J Clin Invest 1986;78:18-25.

13 McFadden ER Jr, Ingram RH Jr. Exercise-induced airway obstruction. Annu Rev Physiol 1983;45:453-63.

14 Anderson SD. Recent advances in the understanding of exercise-induced asthma. Eur J Respir Dis 1983; 225(suppl 128):225-31.

15 Mathew OP, Renners JE. Respiratory function of the upper airway. In: Saunders NA, Sullivan CE, eds. Sleep and breathing. New York: Dekker, 1984:163-200.

16 Takagi Y, Proctor DF, Salman S, Evering S. Effects of cold air and carbon dioxide on nasal air flow resistance. Ann Otol Rhinol Laryngol 1969;78:40-8. 